\title{
Eye Rubbing, a Sine Qua Non for Keratoconus?
}

Damien Gatinel

\begin{abstract}
Keratoconus, a dystrophy of unknown origin, remains an ophthalmic enigma. The contrast between the presence of marked structural changes and deformation of the corneal wall and the relative absence of specific genetic and biomolecular findings continues to intrigue ophthalmologists. In Marfan syndrome, where genetic and molecular abnormalities are well identified, and similar changes in collagen observed, the cornea tends not to be steeper, irregular or ectatic, but are globally flatter. This suggests that an external mechanical force may be necessary to induce the apparition and progression of the ectatic process in keratoconus. Eye rubbing has long been acknowledged as a risk factor for keratoconus and its progression, but could it in fact be the root cause? Many clinical observations and reports support the hypothesis of eye rubbing as a first and necessary hit for inducing progressive ectatic deformation of the corneal wall. Validating or refuting this hypothesis on the basis of patient admission may be impossible. It is difficult to document the frequency, duration and intensity of eye rubbing in patients with keratoconus, and virtually impossible to prove that every patient who denies the habit truly does not rub his eyes. Both the increase in incidence of atopy and the time spent in front of the computer screen in the general population may account for an increased tendency for eye rubbing, and lead to the perceived increased prevalence of keratoconus in both urban and non-urban areas. This paper explores the possibility that the mechanical stress imposed on the cornea by rubbing may not be as much a second hit evoking the structural changes of a predisposed cornea exhibiting unknown collagen progressive alteration, but rather the necessary trigger and sine qua non of the keratoconic process. Even if this provocative hypothesis is impossible to prove, it is equally difficult to refute, and acknowledging eye rubbing as a possible root cause increases awareness within the general population and if true, could dramatically reduce the incidence of keratoconus, and halt its progression in eyes already affected.
\end{abstract}

Keywords: Computer vision syndrome, Corneal biomechanics, Crosslinking, Ectasia, Eye rubbing, Etiology of keratoconus, Keraotoconus, Marfan syndrome.

How to cite this article: Gatinel D. Eye Rubbing, a Sine Qua Non for Keratoconus? Int J Kerat Ect Cor Dis 2016;5(1):6-12.

Source of support: I thank Cordelia Chan, MD, for assisting the revision of the manuscript.

Conflict of interest: None

\section{INTRODUCTION}

"When the wise man points at the Moon, the idiot looks at the finger." This famous quote is attributed to Confucius

\footnotetext{
Assistant Professor

Department of Anterior Segment and Refractive Surgery Rothschild Ophthalmic Foundation, Paris, France

Corresponding Author: Damien Gatinel, Fondation Rothschild, CEROC, 25 Rue Manin, Paris, France, Phone: +33148036482, e-mail: gatinel@gmail.com
}

and should encourage every clinician and researcher to look beyond the realm of existing evidence when facing the challenging mysteries of various diseases.

Keratoconus (KC) has long fascinated ophthalmologists, and its etiology has remained an enigma since this disease was first characterized in the mid19th century. As an ophthalmologist whose practice is focused on KC detection and management, I have been continually intrigued by the pathophysiology of such a mysterious disease, with the exact mechanism of its hallmark characteristics of progressive thinning and deformation still not fully understood. Ironically, to better understand $\mathrm{KC}$, it may be necessary to forsake the Confucius doctrine and focus our attention literally on the patient's fingers. In doing so, we may better understand the innate mechanism of induction and progression of this enigmatic disease. This shall be the focal point of my article.

"Keratoconus" refers to the abnormal conical shape of the corneal contour, which steepens centrally and flattens peripherally. Slit lamp findings of stromal thinning, scarring, and macroscopically detectable deformation of the corneal dome giving rise to Munson's sign are frequently encountered in patients with a long documented history of KC. A "cone" is a mathematical figure that is infinitely steep at its apex and completely flat on its envelope. Hence, "keratoconus" is merely a descriptive term, which fails to detail the underlying mechanism that results in this unique change to the corneal shape.

Through the many years of my clinical practice, I have encountered seemingly countless patients with $\mathrm{KC}$, either newly diagnosed or with long-standing disease. In my practice, men seem slightly more affected than women, but the age at which the disease is discovered is quite variable, as is the stage of the disease at the time of diagnosis. It is not rare to observe large differences between the right and left eyes of the same patient.

Several years ago, as I was examining a patient with a severe form of the disease, where the corneas were so scarred and protruding that there was no need of a slit lamp exam to raise the suspicion of $\mathrm{KC}$, a question inadvertently arose in my mind, as if I was looking for answers to the enigmatic origin of $\mathrm{KC}$ for the first time: I suddenly wondered how a quiescent organ such as the cornea could undergo such a dramatic morphological change, when there were so few histopathological findings and no real established biological cascade to explain it? 
It occurred to me then that it may be necessary to look at $\mathrm{KC}$ with a fresh eye and from a different perspective, perhaps with the mind of a naïve medical student or a non-ophthalmologist, in order to better understand the disease.

Medical students are usually taught that $\mathrm{KC}$ is a noninflammatory corneal dystrophy, for which the cause remains unknown, but may be related to some yet to be identified inherited collagen abnormality. Yet, there are no specific histological abnormalities to support this theory. Analysis of corneal buttons harvested during full or partial thickness penetrating keratoplasties for advanced $\mathrm{KC}$ is quite disappointingly unremarkable with respect to the microstructure of the corneal collagen microfibrils and keratocytes. Stromal thinning has been recently associated with the number of breaks in Descemet's membrane and thickening of the epithelium with breaks in Bowman's layer. ${ }^{1}$ Certainly, collagen fiber disorganization and various inflammatory markers and enzymes have been documented and identified in harvested corneal buttons, but these alterations correspond to nonspecific tissue disorganization at a microscopic level, and do not really explain the primary pathology leading to the dramatic morphological changes observed in advanced keratoconic corneas. ${ }^{2}$ It is known that a complex regular intertwining of the stromal extracellular matrix is critical in ensuring corneal transparency, ${ }^{3}$ as well as in establishing the appropriate biomechanics required to maintain the corneal shape. ${ }^{4}$ Keratocytes control the synthesis of extracellular matrix components, and mutations or deficiencies in stromal regulatory molecules have been identified in stromal dystrophies, but again there are no such clear and specific changes in corneas with $\mathrm{KC}$.

In contrast, other diseases of a similar scale have pronounced histological changes that correlate well with its macroscopic tissue alterations. In dermatologic diseases, histological findings are eloquent, and they often reveal some obvious cellular and/or extra cellular matrix abnormalities that lead to changes in skin consistency, color, and elasticity. Enzymatic cascades and pathophysiological mechanisms are well established, and the causes of these diseases are usually genetically identified. The same applies to cardiovascular diseases where tissues of the heart or valves exhibiting some functional alteration and/or macroscopic changes have correspondingly well-documented histological, genetic, or metabolic abnormalities.

In this context, the observed cornea changes in Marfan syndrome make a pertinent and compelling comparison with KC. In Marfan syndrome caused by mutations in the FBN1 gene, ${ }^{5}$ connective tissue alterations in cardiac as well as ocular tissues are well documented. The fibrillin gene (FBN1) is located on chromosome 15q15-21,1-4 and encodes for fibrillin-1, a 350-kDa acidic glycoprotein, which is a major component of the 10- to $12-\mathrm{nm}$ extracellular microfibrils. It is known to play a critical role in the strength and elasticity of ocular connective tissues. Corneal biomechanics are therefore significantly altered in patients with Marfan syndrome, and ectopia lentis is also observed. ${ }^{6}$ Interestingly, despite the connective disease, the corneas of patients with Marfan syndrome tend to be flatter ${ }^{7,8}$ and thinner ${ }^{8}$ but do not show ectatic or keratoconic changes. ${ }^{7-9}$ However, abnormal keratometry and pachymetry were both found to be highly associated with ectopia lentis. ${ }^{8}$ Thus, morphologic abnormalities resulting from mutations in fibrillin- 1 are able to produce dislocation of the lens but fail to produce any other corneal topographic abnormalities other than flattening and thinning. This suggests that without an additional factor, altered biomechanics from collagen abnormalities alone may not be sufficient to account for the steepening, weakening, and protrusion seen in cornea ectasia and $\mathrm{KC}$.

With so much focus at the microscopic level yielding little evidence for $\mathrm{KC}$, perhaps the scene of investigation should be "zoomed out" and enlarged to the macroscopic world, in particular to a "force" that could invoke the keratoconic disease and explain the progressive thinning and deformation of the cornea. Naturally, chronic and vigorous eye rubbing comes to mind.

A positive correlation between eye rubbing and aggravation of KC has been described, ${ }^{9-13}$ but let us look at this from a different perspective. Could eye rubbing alone, or any other repeated traumatic force exerted on the corneal wall, give rise to the characteristic changes in $\mathrm{KC}$ ? Could this mechanical trauma be the only reason for $\mathrm{KC}$ to occur and exist?

I have long been intrigued with the hypothesis that KC may never occur in a cornea that is not subjected to repeated mechanical trauma, such as with vigorous eye rubbing. In science, any hypothesis deserves a critical eye and examination before being validated and accepted. A scientific mind may be particularly reluctant to consider any tentative explanations valid, especially when they are as simplistic as eye rubbing. Given the work and efforts that have been dedicated to determining the cause(s) of $\mathrm{KC}$, this simple hypothesis even seems provocative. That eye rubbing is the cause of $\mathrm{KC}$ is indeed too straightforward and simple an assertion, but it deserves legitimate attention. If valid, this hypothesis can have profound implications, as suppression of this habit would ultimately lead to the stabilization or even disappearance of the disease that has stumped ophthalmologists around the world.

One interesting way to evaluate this hypothesis is to verify its compatibility with what is commonly known about KC. 
Firstly, let us look at the genetics of KC. One can argue that $\mathrm{KC}$ has a genetic basis, but KC has been found to have a poorly defined pattern of inheritance. ${ }^{14}$ If the occurrence of the disease were a result of genetic predisposition alone, why would such a low percentage of patients report awareness of other family members with this condition? ${ }^{15,16}$ Could KC require an interaction with environmental factors to make a genetically determined predisposition manifest as a phenotypically apparent disease? This is where eye rubbing may come into play. ${ }^{9}$ It is possible that a predisposition to corneal changes induced by eye rubbing may account for some genetic modulation of the disease expression. Examples of genetic conditions where eye rubbing and $\mathrm{KC}$ are strongly associated are in Down's syndrome, ${ }^{17,18}$ Tourette, ${ }^{19,20}$ and atopic disease. While not every atopic or patient with intellectual disability may be an eye rubber, $\mathrm{KC}$ often occurs in these patients who exert excessive chronic or seasonal compression on the cornea. The association between $\mathrm{KC}$ and atopy does not seem to be influenced by the type of allergy, e.g., hay fever or vernal keratoconjunctivitis, but rather by the extent of repeated mechanical trauma induced by rubbing the eye. Subtle differences between "eye rubbing" of allergic and KC patients have been reported, ${ }_{1}^{13}$ but in essence, the fact that $\mathrm{KC}$ patients rub their eyes quite vigorously may indicate that this repetitively applied force is the direct cause of the corneal deformation, regardless of the cause of eye rubbing itself. Some patients combine various techniques of eye rubbing, using both their finger pulps and knuckles consecutively. (See video https://www.youtube.com/watch?v=9Hzq B9ZwHto.)

Eye rubbing facilitates lubrication, provides immediate comfort and relief, but also leads to the occurrence of a central corneal epitheliopathy, leading to further discomfort, which triggers eye rubbing again. So a vicious cycle ensues.

The correlation between genetics, atopy, and chronic eye rubbing is therefore likely to be an inherited vulnerability or susceptibility accelerating the response to mechanical trauma, giving rise to the biomechanical changes seen in KC. I am inclined to suspect that in the absence of rubbing (or any other cause of local repeated biomechanical stress), the chances of developing a KC or corneal ectasia pattern may be very low or even nonexistent, but this is difficult to validate. Nevertheless, explaining to young allergic patients and their parents (as well as to refractive surgery candidates) that eye rubbing should be banished from their habits is mandatory. In addition, adequate environmental solutions aimed at reducing pollution, irritants, and allergies are necessary complementary actions that will hopefully help reduce the incidence and severity of $\mathrm{KC}$.
There is a perception that $\mathrm{KC}$ is less prevalent among patients with advancing age. ${ }^{21,22}$ Keratoconus is perceived as a "young person's disease." This perception may partly result from a bias, due to the increasingly early $\mathrm{KC}$ detection in the younger population of refractive surgery candidates screened with corneal topography in routine eye examinations. Nevertheless, the reason for a reduction in the prevalence of the disease with advancing age remains a challenge to clinicians and researchers. While KC has been reported to be associated with diseases that increase mortality such as obstructive apnea syndrome, ${ }^{23}$ the difference between the prevalence of $\mathrm{KC}$ in the younger population compared with the older population may in fact be a reflection of a dramatic increase in environmental conditions that evoke an increase in eye rubbing. In the last decades, the prevalence of atopy has dramatically increased, ${ }^{24}$ possibly in tandem with environmental changes such as high levels of air pollution in urban areas and Western lifestyle. ${ }^{25}$ This increase in the prevalence of atopy and the subsequent eye rubbing it incites may explain the higher incidence of $\mathrm{KC}$ in the younger population, creating the relative perception that the disease is less prevalent in the older patients. Computer vision syndrome (CVS) is a collection of symptoms related to prolonged work at a computer display. Symptoms reported by computer users associate internal ocular symptoms (strain and ache), and external ocular symptoms such as dryness, irritation, and burning. ${ }^{26}$ Eye rubbing may be more pronounced and frequent in the population suffering from the ocular consequences of extended computer use. With many school-age kids and even preschoolers spending hours in front of a computer every day, it's worth considering that computers and digital screens might trigger frequent eye rubbing and also account to the incidence of KC.

Patients who rub their eyes with their knuckles are those in whom KC seem to occur more frequently. The knuckles are the most rigid part of the fingers, much harder than the finger's pulp. To the scale of the cornea, which can be described as a dome of tissue of about half a millimeter thick centrally and consisting of about 250 collagen fibers of $2-\mu \mathrm{m}$ diameter, the force exerted by the fingers and knuckles do represent significant mechanical stress. Some authors have proposed that the biomechanical modification encountered in KC corneas is focal in nature, rather than a uniform generalized weakening. ${ }^{27}$ They proposed the concept that a focal reduction in the elastic modulus precipitates a cycle of biomechanical decompensation, driven by asymmetry in the biomechanical properties. A repeating cycle of increased strain and stress redistribution would then lead to subsequent focal steepening and thinning. It seems to me that this localized, rather than global, alteration 
in the corneal biomechanics is compatible with the eye rubbing theory, with repeated eye rubbing inducing the focal steepening and thinning pathognomonic of $\mathrm{KC}$.

How does eye rubbing cause $\mathrm{KC}$ at a microscopic level? Eye rubbing for 60 seconds has been showed to increase the level of tear matrix metalloproteinase 13 , interleukin 6, and tumor necrosis factor $\alpha{ }^{28}$ These upregulated proteases and inflammatory molecules may be causal links between eye rubbing and KC. They may account for the apparent lack of inflammation in $\mathrm{KC}$. However, the absence or little evidence of histological findings of inflammation, such as neovascularization or cell infiltration, strongly suggests that $\mathrm{KC}$ is not primarily caused by an inflammatory process. Eye rubbing may lead to the progressive distention and severing of the corneal collagen fibrils. Disorganization of the arrangement of the collagen fibers consequent to the excessively strong compressing effect of the fingers would disturb the homogeneity of the fiber matrix, which in normal corneas helps to dissipate the mechanical energy evenly and maintains the regularity of the corneal curvature. By creating one or several localized weakened zones within which the force exerted by the intraocular pressure causes deformation of the corneal wall, chronic eye rubbing would thus induce a change in corneal macroscopic regularity and lead to the classic topographical features of KC. For the same eye rubbing intensity and frequency, eyes with thin corneas (less collagen fibrils) may develop a KC pattern sooner than eyes with thicker corneas. The quality or mechanical strength of the collagen fibrils may also influence the susceptibility to developing an ectatic pattern.

The frequency and duration of eye rubbing in patients may be difficult to document, and is likely to be underestimated. The reported prevalence of eye rubbing is between 66 and $80 \% .{ }^{29,30}$ However, in another study, $46 \%$ of patients diagnosed with $\mathrm{KC}$ denied rubbing their eyes vigorously. ${ }^{31}$ This contrasts with the conclusion of a study that involved a multivariate analysis of risk factors for $\mathrm{KC}$ disease, in which eye rubbing was found to be the only significant predictor. ${ }^{32}$ In two recent studies of KC occurring in children as young as 4 years of age, eye rubbing seemed to be the causative factor. ${ }^{33,34}$ In addition, eye rubbing was found in $91.84 \%$ of children with $\mathrm{KC}$ in an epidemiological study. ${ }^{35}$

When first asked about repetitive eye rubbing, KC patients often underreport this habit. Some of the deniers have been caught rubbing their eyes while interviewed, while a large proportion of them are not even conscious of their habit and will only realize it when they are back home after having been sensitized to the point. It is important to elicit a history of eye rubbing from patients in an appropriate manner. Often, these patients are too embarrassed to admit to this habit and may not readily volunteer the information. The physician should first put the patient at ease, especially at the first visit. Asking family, friends, or coworkers to survey and observe is often rewarding: $\mathrm{KC}$ patients often report their eye rubbing more accurately when they return for a follow-up visit. I have received numerous emails from newly diagnosed patients who realized after their first consultation that they rub their eyes far more frequently than previously thought.

Patients should be asked to pay attention to specific time periods of eye rubbing such as after awakening, before sleeping, or after removal of their contact lenses. In patients who admit to rubbing, the worse (more keratoconic) eye is often the eye which is more often rubbed. ${ }^{10}$ In itself, this factor suggests a strong link between eye rubbing and KC. I have seen cases of what seems to be a true unilateral $\mathrm{KC}$ where the patient only rubs the affected eye, and the contralateral "healthy" unrubbed eye shows no topographical abnormalities and would subsequently remain negative to all topography and tomography testing, indices, and scores. Although it does not prove that eye rubbing is the inciting cause of the disease, these cases serve as compelling evidence to support the definitive association between eye rubbing and $\mathrm{KC}$.

I have followed patients in whom $\mathrm{KC}$ appeared unusually late in life (between 30 and 40 years of age). In each of these cases, sudden changes in professional and/or living conditions resulted in triggers for intense and chronic eye rubbing. I remember the case of a 28-year-old male, without any remarkable ophthalmic or general medical history, who had to shift from day to night maintenance work in a factory. Working at night caused the patient to be chronically fatigued, and he spontaneously admitted to commencing rubbing his eyes circularly with his knuckles. Another patient started to work as a baker at the age of 33 and developed chronic ocular irritation due to the projection of wheat dust into his surroundings, and he too started to rub his eyes. Two years later, he complained of a decrease in vision and developed astigmatism. A corneal topography examination revealed the presence of early $\mathrm{KC}$, requiring the adaptation of rigid gas permeable contact lenses. A third patient had to move from Paris to a provincial area at the age of 36, where he developed severe asthma and pollen-related allergic conjunctivitis. This made him rub his eyes quite frequently. Despite an otherwise unremarkable ophthalmic history, he developed KC two years later.

These patients were all initially spectacle free without any familial history of KC, before changes in their lifestyle 
and habits occurred. They claimed to have "excellent vision" until they started to rub their eyes repeatedly. At some point in time after the beginning of their chronic eye rubbing habits, they noticed a progressive decrease in their visual acuity, which would eventually affect both eyes, but first occurring in the eye that they would rub most. In my experience, the time between the onset of $\mathrm{KC}$ and the initiation of chronic eye rubbing is usually about 2 to 3 years. Children who start to rub their eyes by the age of 10 to 12 years develop $\mathrm{KC}$ when they are teenagers.

Of course, I have encountered patients who deny rubbing their eyes repeatedly, visit after visit. However, there may be another mechanical contribution to the occurrence of a KC pattern in these patients. Sleeping on the side with a pillow or hand exerting a direct pressure or compression on the eyelids could be the causative mechanical stress factor for the onset of KC. Excess fluid within the corneal stroma, as a result of low overnight oxygen levels, may make the cornea more vulnerable. The cumulative effect of these forces, exerted night after night, may alter corneal biomechanics and lead to a particular topographic pattern of KC characterized by marked inferior steepening with decentration of the apex, which is unilateral (if the patient always sleeps on the same side) or bilateral (if the patient sleeps alternately on both sides) (Fig. 1). This sleeping habit may also induce floppy eyelids, which could explain its association with KC. ${ }^{36}$ This tendency has been coined "pillow hugging" and was previously identified as a cause of $K C{ }^{13}$ Unlike what is commonly observed in eye rubbers, the corneal thickness of these "nightly compressed" eyes is preserved, and isolated mild to pronounced peripheral steepening (which is inferior in most cases) may constitute the main topographic feature. Hence, the pertinence of labeling theses eyes as "keratoconic" may be debated.

I have also encountered the same rubbing tendencies in a number of patients with post-LASIK keratectasia. In particular, mysterious ectasia cases where no obvious sign of early subclinical KC preoperatively could be identified could perhaps be caused by eye rubbing. Post-LASIK epitheliopathy and dry eye are known to trigger eye rubbing in some patients. In one observation, cessation of eye rubbing sufficed to stabilize an early progressive ectatic pattern.

Abnormal corneal topography is considered the main risk factor for the development of post-LASIK keratectasia. Eye rubbing could very well be the cause of these abnormal topography patterns detected at the time of preoperative evaluation. In many situations where I found a suspicious topography pattern ranking from "funny looking" to early $\mathrm{KC}$, chronic eye rubbing was acknowledged by the patient.

In summary, when susceptible eyes undergo some level of chronic rubbing, patients can develop a condition characterized by progressive corneal deformation and thinning, which we currently call "KC." Genetic or surgical conditions (e.g., LASIK) can predispose or accelerate the mechanical weakening effect of chronic eye rubbing. Despite the disappointing simplicity of this theory, especially to those who seek to verify the gene or metabolic cause of $\mathrm{KC}$, the hypothesis that chronic eye rubbing is the sine qua non for $\mathrm{KC}$ remains compatible with most clinical observations and current scientific data. Unfortunately, non-refutation does not suffice to prove the theory. However, considering this
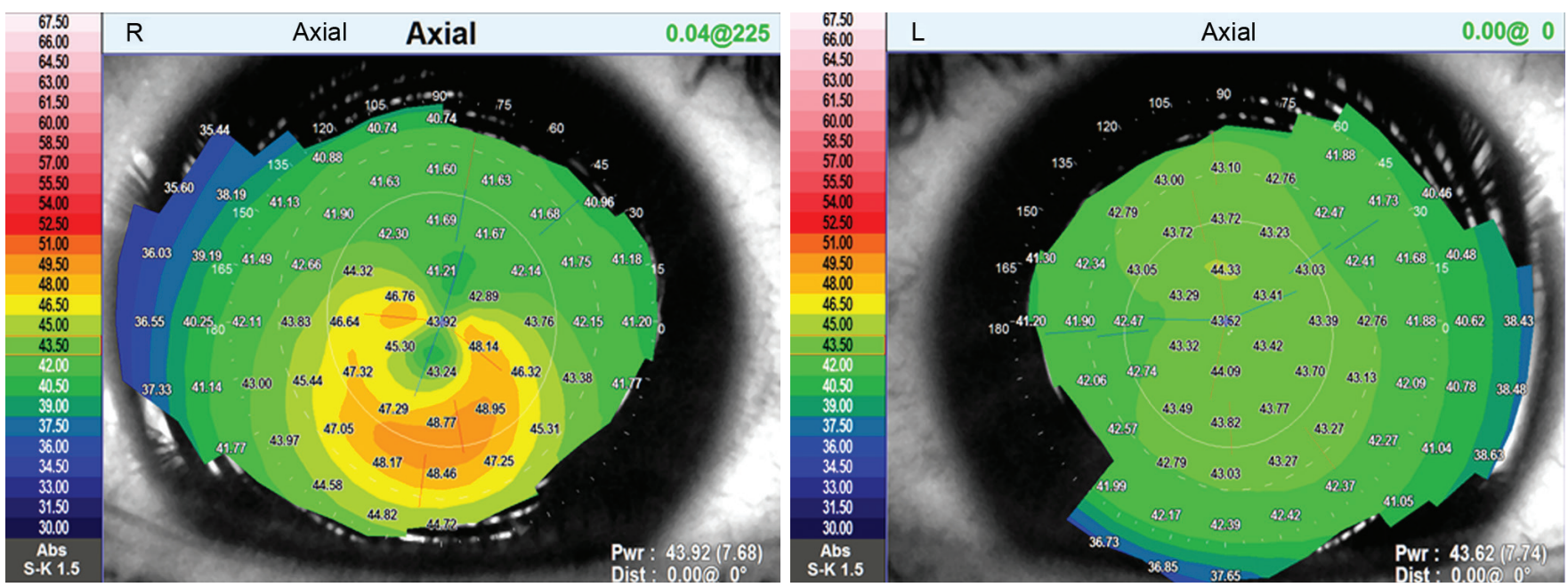

Fig. 1: Topographical aspect of a "unilateral" keratoconus in the right eye (axial map, OPDscan III) in a 34-year-old patient who denies rubbing his eyes vigorously. The corneal pachymetry was $550 \mu \mathrm{m}$ in both eyes centrally. However, the patient admitted sleeping on the right side, with the pillow placed under his head and applied to the right side of his face by his right hand. During a subsequent visit, the patient recognized that he realized that he often rubbed his eyes, once having been warned of this eventuality. The combination of repeated daytime rubbing and nighttime compression of the right eye's cornea may explain the increased severity of its topographic deformation 
hypothesis valid can have positive consequences, as it could lead to the possibility of lowering the incidence of, if not eradicating, $\mathrm{KC}$.

I have written this article not only to draw the attention of my colleagues to eye rubbing but also to alert patients and their relatives to the consequences of this habit. It does seem to me that it is worth tentatively accepting this correlation, and it would be very interesting to monitor the incidence of $\mathrm{KC}$ in a population exempt from any eye rubbing. If eye rubbing is not the cause of $\mathrm{KC}$, it has certainly been implicated in its progression. Hence, cessation of eye rubbing is important, as it may be sufficient to halt the evolution and progression of KC and avoid interventional procedures on the cornea, such as the oxidative process of riboflavin stromal soaking and artificial ultraviolet light exposure, which is the basis of the technique of corneal cross-linking, ${ }^{37}$ believed to effect corneal collagen reticulation and anterior corneal strengthening. ${ }^{38}$ Eliminating excessive eye rubbing during childhood and the teenage years could even result in the eradication of this disease. While this goal may seem unrealistic and unattainable (as it is virtually impossible to stop everyone from rubbing their eyes), increasing the awareness of this causative role may help to decrease the incidence of KC more efficiently than anything else.

In conclusion, there is sufficient evidence to suggest that the etiology of KC has a strong mechanical dimension. The hypothesis of eye rubbing being the sine qua non for KC may be difficult to prove, but with current evidence, both scientific and anecdotal, this hypothesis may be equally difficult to refute. It is therefore worth sharing, so spread the word ... Do not rub your eyes, and stop your loved ones from doing so!

\section{REFERENCES}

1. Sykakis E, Carley F, Irion L, Denton J, Hillarby MC. An in depth analysis of histopathological characteristics found in keratoconus. Pathology 2012 Apr;44(3):234-239.

2. Cheung IM, McGhee CN, Sherwin T. A new perspective on the pathobiology of keratoconus: interplay of stromal wound healing and reactive species-associated processes. Clin Exp Optom 2013 Mar;96(2):188-196.

3. Maurice DM. The structure and transparency of the cornea. J Physiol 1957 Apr 30;136(2):263-286.

4. Müller LJ, Pels E, Vrensen GF. The specific architecture of the anterior stroma accounts for maintenance of corneal curvature. Br J Ophthalmol 2001 Apr;85(4):437-443.

5. Groth KA, Hove H, Kyhl K, Folkestad L, Gaustadnes M, Vejlstrup N, Stochholm K, Østergaard JR, Andersen NH, Gravholt $\mathrm{CH}$. Prevalence, incidence, and age at diagnosis in Marfan Syndrome. Orphanet J Rare Dis 2015 Dec 2;10:153.

6. Kara N, BozkurtE, Baz O, Altinkaynak H, Dundar H, YukselK, Yazici AT, Demirok A, Candan S. Corneal biomechanical properties and intraocular pressure measurement in Marfan patients. J Cataract Refract Surg 2012 Feb;38(2):309-314.
7. Maumenee IH. The cornea in connective tissue diseases. Ophthalmology 1978 Oct;85:1014-1017.

8. SultanG,Baudouin C,AuzerieO, DeSaintJean M, GoldschildM, Pisella PJ; Marfan Study Group. Cornea in Marfan disease: Orbscan and in vivo confocal microscopy analysis. Invest Ophthalmol Vis Sci 2002 Jun;43(6):1757-1764.

9. Maumenee IH. The eye in the Marfan syndrome. Trans Am Ophthalmol Soc 1981;79:684-733.

10. McMonnies CW. Mechanisms of rubbing-related corneal trauma in keratoconus. Cornea 2009 Jul;28(6):607-615.

11. McMonnies CW, Boneham GC. Keratoconus, allergy, itch, eye-rubbing and hand-dominance. Clin Exp Optom 2003 Nov;86(6):376-384.

12. Sugar J, Macsai MS. What causes keratoconus? Cornea 2012 Jun;31(6):716-719.

13. Carlson AN. Expanding our understanding of eye rubbing and keratoconus. Cornea $2010 \mathrm{Feb} ; 29(2): 245$.

14. Burdon KP, Vincent AL. Insights into keratoconus from a genetic perspective. Clin Exp Optom 2013 Mar;96(2):146-154.

15. Kennedy RH, Bourne WM, Dyer JA. A 48-year clinical and epidemiologic study of keratoconus. Am J Ophthalmol 1986 Mar 15;101(3):267-273.

16. Rabinowitz YS. Keratoconus. Surv Ophthalmol 1998 Jan-Feb; 42(4):297-319.

17. Scherbenske JM, Benson PM, Rotchford JP, James WD. Cutaneous and ocular manifestations of Down syndrome. J Am Acad Dermatol 1990 May;22(5 Pt 2):933-938.

18. van Splunder J, Stilma JS, Bernsen RM, Evenhuis HM. Prevalence of ocular diagnoses found on screening 1539 adults with intellectual disabilities. Ophthalmology 2004 Aug;111(8):1457-1463.

19. Kandarakis A, Karampelas M, Soumplis V, Panos C, Makris N, Kandarakis S, Karagiannis D. A case of bilateral self-induced keratoconus in a patient with Tourette syndrome associated with compulsive eye rubbing: case report. BMC Ophthalmol 2011 Sep 21;11:28.

20. Mashor RS, Kumar NL, Ritenour RJ, Rootman DS. Keratoconus caused by eye rubbing in patients with Tourette Syndrome. Can J Ophthalmol 2011 Feb;46(1):83-86.

21. McMonnies CW. Quo vadis older keratoconus patients? Do they die at younger ages? Cornea 2013 Apr;32(4):496-502.

22. Carlson AN. Where are the older patients with keratoconus? Cornea 2010 Apr;29(4):479-480.

23. Gupta PK, Stinnett SS, Carlson AN. Prevalence of sleep apnea in patients with keratoconus. Cornea 2012 Jun;31(6): 595-599.

24. Sibbald B, Rink E, D'Souza M. Is the prevalence of atopy increasing? Br J Gen Pract 1990 Aug;40(337):338-340.

25. Herbert OCh, Barnetson RS, Weninger W, Krämer U, Behrendt $\mathrm{H}$, Ring J. Western lifestyle and increased prevalence of atopic diseases: an example from a small Papua New Guinean island. World Allergy Organ J 2009 Jul;2(7):130-137.

26. Klamm J, Tarnow KG. Computer Vision Syndrome: A Review of Literature. Medsurg Nurs 2015;24(2):89-93.

27. Roberts CJ, Dupps WJ Jr. Biomechanics of corneal ectasia and biomechanical treatments. J Cataract Refract Surg 2014 Jun;40(6):991-998.

28. Balasubramanian SA, Pye DC, Willcox MD. Effects of eye rubbing on thelevels of protease, proteaseactivity and cytokines in tears: relevance in keratoconus. Clin Exp Optom 2013 Mar; 96(2):214-218.

29. Coperman PWM. Eczema and keratoconus. Br Med J 1965 Oct 3;2(5468):977-979. 
30. Karseras AG, Ruben M. Aetiology of keratoconus. Br J Ophthalmol 1976 Jul;60(7):522-525.

31. Zadnik K, Barr JT, Edrington TB, Everett DF, Jameson M, McMahon TT, Shin JA, Sterling JL, Wagner H, Gordon MO. Baseline findings in the Collaborative Longitudinal Evaluation of Keratoconus (CLEK) Study. Invest Ophthalmol Vis Sci 1998 Dec;39(13):2537-2546.

32. Bawazeer AM, Hodge WG, Lorimer B. Atopy and keratoconus: a multivariate analysis. Br J Ophthalmol 2000 Aug;84(8): 834-836.

33. Gunes A, Tok L, Tok Ö, Seyrek L. The youngest patient with bilateral keratoconus secondary to chronic persistent eye rubbing. Semin Ophthalmol 2015 Dec;30(5-6):454-456.

34. Panahi-Bazaz MR, Sharifipour F, Moghaddasi A. Bilateral keratoconus and corneal hydrops associated with eye rubbing in a 7-year-old girl. J Ophthalmic Vis Res 2014 Jan;9(1): 101-105.

35. Léoni-Mesplié S, Mortemousque B, Mesplié N, Touboul D, Praud D, Malet F, Colin J. Epidemiological aspects of keratoconus in children. J Fr Ophtalmol 2012 Dec;35(10):776-785.

36. Gordon-Shaag A, Millodot M, Shneor E, Liu Y. The genetic and environmental factors for keratoconus. Biomed Res Int 2015:795-738.

37. Kanellopoulos AJ, Moustou V, Asimelis G. Evaluation of visual acuity, pachymetry and anterior surface irregularity in keratoconus and crosslinking intervention follow-up in 737 cases. J Kerat Ect Cor Dis 2013;2(3):95-103.

38. Gatinel D. Effectiveness of corneal collagen crosslinking in vivo for corneal stiffening. J Cataract Refract Surg 2014 Nov;40(11):1943-1944. 